\title{
ALTERAÇÕES FUNCIONAIS DE UM GRUPO DE IDOSAS DURANTE A HOSPITALIZAÇÃO E NA ALTA HOSPITALAR
}

[Functional alteration presented by a group of old women in the hospitalization and at the discharge]

\author{
Maria José Sanches Marin* \\ Emília Luígia Saporiti Angerami**
}

RESUMO: Os idosos, freqüentemente, apresentam alterações específicas e são vulneráveis a múltiplas perdas, principalmente das funções do organismo, predispondo-os a doenças e a hospitalização. Considerando que os idosos, quando hospitalizados, apresentaram alteração aguda no seu estado de saúde, que a baixa reserva funcional dificulta o seu restabelecimento e que os cuidados a esses indivíduos devem ser contínuo, no presente estudo identificou-se as alterações funcionais apresentadas por um grupo de 50 idosas internadas em Unidade de Clínica Médica de um Hospital Geral, durante a hospitalização e na alta hospitalar. Na análise dos resultados constatou-se altas taxas de alterações das funções, no momento da admissão, muitas das quais foram apenas amenizadas durante a hospitalização e outras se modificaram. Entre as alterações funcionais apresentadas pelas idosas destacam-se aquelas referentes à ingestão/ digestão presentes em 35 (70\%) delas durante a internação e em 32 (64\%) na alta hospitalar, alterações de pele/cabelo/ unhas em 33 (66\%) das idosas durante a hospitalização e em 31 (62\%) na alta hospitalar, alterações de movimentação e deambulação em 31 (62\%) e 30 (60\%) delas durante a internação e na alta hospitalar respectivamente, entre outras, como alterações da circulação, visão, eliminações, sono e repouso, sensibilidade, audição e respiração. Além disso, apresentaram em média dois diagnósticos médicos/idosa e faziam uso de 2,6 medicamento/idosa, deixando claro a necessidade de ações capazes de proporcionar continuidade na complexa assistência requerida na promoção, prevenção, cura e reabilitação da saúde dessas pessoas.

PALAVRAS-CHAVE: Idoso; Hospitalização; Alta do paciente.

Doutora em Enfermagem e Docente do Curso de Enfermagem da Faculdade de Medicina de Marília.

** Professora Titular do Departamento de Enfermagem Geral e Especializada da Escola de Enfermagem da Universidade de São Paulo, Ribeirão Preto.

\section{INTRODUÇÃO}

Nossa atuação profissional permite contato freqüente com idosos e a vivência intensiva dos problemas que os envolvem, tanto durante a hospitalização, como na alta hospitalar e no pós-alta.

A preocupação com a velhice vem aumentando no mundo todo, nos últimos decênios, devido à constatação do aumento da média de vida da população, atribuído às melhores condições sanitárias, à profilaxia de doenças, ao surgimento de novas drogas e ao planejamento familiar. (BURNISIDE, 1979; GOMES; FERREIRA, 1985; BEQUÓ; LEITE, 1988).

No Brasil, chama a atenção a modificação da expectativa de vida ao nascer ao longo do século. Em 1900, os indivíduos com mais de 60 anos perfaziam um percentual de $3 \%$ e a expectativa de vida era de 33,7 anos. Em 1970, verificou-se um percentual de $5,2 \%$ e uma expectativa de vida de 57,1 anos, sendo que, no censo de 1980 , esse percentual subiu para $6.5 \%$ e a expectativa de vida, para 63.5. Essa expectativa, em 1990, era de 68,4 anos e as pessoas com mais de 60 anos representavam $8 \%$ da população (IBGE, 1991). Para o ano de 2005, estima-se que a esperança de vida do brasileiro seja de 72 anos e as pessoas com mais de 60 anos perfaçam um total de $15 \%$ da população (GOMES; FERREIRA, 1985; NÉRI, 1993; VERAS,1994).

O processo de envelhecimento é uma realidade sem retrocesso e segundo CHELALA (1992), pode-se considerar país envelhecido aquele que apresenta, na sua população geral, $7 \%$ ou mais de idosos. Essa é uma tendência mundial, destacando-se poucos países que não atingiram esse patamar no ano de 2000.

Juntamente com as modificações na estrutura etária da população, constatam-se mudanças epidemiológicas, com a substituição das causas principais de morte por doenças parasitárias de caráter agudo pelas doenças crônicodegenerativas (diabetes, acidente vascular cerebral, neoplasias, hipertensão arterial, demência senil e outras) que se transformam em problemas de longa duração, interferem 
nas condições de vida e envolvem, para atendimento adequado, grande quantidade de recursos materiais e humanos. (LESSA, 1990).

Entre os fatores que afetam a qualidade de vida dos idosos, as alterações funcionais são bastante freqüentes e desempenham papel fundamental quando se pretende identificar o processo de envelhecimento. Elas ocorrem em todos os sistemas e aparelhos orgânicos, porém, em velocidades distintas em diferentes sistemas do mesmo indivíduo.

As alterações funcionais dos idosos justificam-se pela baixa reserva funcional apresentada por eles, o que os torna mais vulneráveis em situação de estresse orgânico e com maior dificuldade de restabelecer as alterações aguda no estado de saúde.

De acordo com a teoria da hierarquia das necessidades humanas básicas de MASLOW (1982), as necessidades fisiológicas precedem qualquer outra. Portanto, se o paciente apresentar alterações como dor, fadiga, fraqueza, estas necessitam ser satisfeitas em primeiro lugar. No entanto, conforme descrito pelo próprio autor, tal hierarquia não deve ser vista com rigidez, pois fatores do próprio indivíduo podem interferir. Assim, quando se trata do idoso, os aspectos fisiológicos devem ser considerados dentro do seu limiar de capacidade, nem sempre sendo possível deixá-lo em um estado ótimo de bem-estar, uma vez que ele pode ser variável de acordo com cada situação. Além disso, as alterações funcionais não podem ser vistas sem considerar o contexto geral do idoso.

Acredita-se, portanto, que apenas o conhecimento, a experiência profissional e o raciocínio crítico poderão auxiliar na manutenção do equilíbrio ao se proporem soluções quando as necessidades básicas estiverem afetadas.

Às alterações fisiológicas que acontecem com o avanço da idade, faz com que as pessoas envelhecidas, mesmo as que têm boa saúde, se debilitem paulatinamente limitando as funções do organismo, tornando-as cada vez mais predispostas à dependência para a realização do autocuidado, à perda da autonomia e da qualidade de vida. Com isso, também se tornam mais suscetíveis a doenças e, como conseqüência, vem à hospitalização.

O idoso, segundo GUTIERREZ GAMBARO (1987), requer com freqüência a hospitalização basicamente motivada por patologias degenerativas (cardiovascular, acidente vascular cerebral, demências e afecções neoplásicas), patologias agudas (infecções respiratórias, como broncopneumonia, infecções do trato urinário, etc.) e por problemas traumáticos (fraturas em geral).

Tem-se observado que os pacientes recebem alta assim que os problemas mais emergentes são resolvidos devido à necessidade de redução dos custos, à grande demanda de leitos hospitalares e aos riscos que a hospitalização pode causar, retornando para as famílias e para a comunidade ainda com uma gama de problemas, muitas vezes complexos, necessitando de assistência adequada.

MARIN (1995), ao estudar 73 idosos internados em Unidade de Clínica Médica, no momento da alta hospitalar, constatou inúmeros problemas de ordem funcional, ambiental, social e econômico, deixando entrever preocupações intensas e diversas.

Diante de tais indicativos, o presente estudo propõe identificar as alterações funcionais apresentadas por um grupo de idosas durante a hospitalização e na alta hospitalar, com a finalidade de contribuir na formulação de estratégias visando a continuidade do cuidado.

\section{METODOLOGIA}

Trata-se de um estudo descritivo tipo avaliativo, o que para TRIVINOS (1987) permite identificar existência de necessidades e proporcionar dados básicos para futuros estudos ou ações.

O estudo foi realizada na unidade de Clínica Médica e Cirúrgica Feminina (ala D) do Hospital de Clínicas I, da Faculdade de Medicina de Marília. A referida Unidade conta, atualmente, com 16 leitos, destinados ao atendimento de idosas com alterações clínicas e cirúrgicas de urgência e emergência, provenientes, portanto, do pronto socorro.

O estudo contou com a participação de idosas que residiam em Marília e se encontravam internadas na unidade referida acima, no período de abril a junho de 2001 . Foram excluídas do estudo as idosas que se internaram em estado grave, com forte ameaça à vida.

Para realização do estudo, um grupo de 50 idosas foi acompanhado desde o momento da internação, onde se implementou a coleta de dados, até 48 horas após a admissão, conforme roteiro previamente estabelecido (anexo I), que contém os dados de identificação e os dados funcionais (ingestão/digestão, respiração, circulação, eliminação/urinária, eliminação/fecal, eliminação/secreção, pele/cabelo/unhas, sensibilidade/regulação, sentido, sono/ repouso, movimentação/deambulação, estado mental).

De acordo com os dados coletados implementou-se o planejamento da assistência de enfermagem o que permitiu o acompanhamento das mesmas durante todo o processo de hospitalização e a identificação de seus problemas na alta hospitalar.

Para efetivar a coleta de dados foi solicitada autorização à comissão de ética da Faculdade de Medicina de Marília, a qual manifestou-se favorável à mesma. 
Antes de iniciar a coleta de dados as idosas e/ou cuidadores foram orientados quanto à finalidade, ao procedimento e informados de que estariam livres para participar ou não do estudo. Quando houve interesse em participar, solicitou-se a assinatura da pessoa para o termo de consentimento livre e esclarecido.

\section{RESULTADOSE DISCUSSÕES}

Entre as idosas em estudo, 13 (26\%) eram pertencentes à faixa etária dos 70 a 74 anos, 12 (24\%) na faixa etária entre 60 e 64 anos, dez (20\%) na faixa etária entre 65 e 69 anos, sendo que apenas quatro (8\%) delas tinham 90 anos ou mais. A faixa de idade a que o idoso pertence tem sido vista na literatura como um aspecto importante a considerar devido ao maior risco de adoecer e de apresentar maior grau de dependência, quanto mais avançada ela se apresenta.

Quanto ao grau de comunicação, 35 (70\%) das idosas apresentaram-se em boas condições de comunicação verbal, enquanto cinco delas (10\%) eram pouco comunicativas. Ainda cinco (10\%) das idosas eram confusas e outras cinco (10\%) não apresentavam condições de comunicação verbal, devido à demência senil ou a conseqüências de alterações orgânicas diversas comuns entre elas.

Quanto à escolaridade, 31 (62\%) das idosas eram analfabetas, 16 (32\%) tinham o primeiro grau incompleto e apenas três (6\%) tinham o primeiro grau completo.

As cinqüenta idosas do estudo apresentaram, registrados nos prontuários, noventa e oito diagnósticos, com uma média de dois diagnósticos por idosa. As patologias mais incidentes entre elas referem-se àquelas do sistema cardiocirculatório 38 (38,8\%). A estas seguem-se as doenças das glândulas endócrinas, nutrição e metabolismo 12 $(12,2 \%)$, as do sistema respiratório nove $(9,2 \%)$, além das doenças do sistema osteoarticular e tecido conjuntivo 11 $(11,2 \%)$, seguidas por doenças da pele e tecido celular subcutâneo nove $(6,1 \%)$, entre outras. Além dessas patologias é possível a existência de outras que não estavam registradas no prontuário.

Os medicamentos utilizados pelas idosas foram agrupados de acordo com o principal mecanismo de ação, verificou-se que os mais receitados são os hipotensores 22 $(16,9 \%)$, em seguida vêm os antibióticos $13(10 \%)$, hipoglicemiantes $11(8,5 \%)$, diuréticos dez (7,7\%), anticoagulantes plaquetários oito $(6,2 \%)$, cardiotônico seis $(4,6 \%)$, entre outros. Para as 50 idosas do estudo foram receitados cento e trinta medicamentos, em média 2,6 medicamento/idosa.
No gráfico 1, constam os dados das alterações relacionadas com a biologia da idosa durante a internação e na alta hospitalar. Ao se referir às alterações de ingestão e digestão, encontradas durante a internação, constata-se que, em 35 (70\%), havia ocorrência de obesidade, prótese mal adaptada, alta ingestão de carboidratos, uso de sonda nasogástrica, ausência de dentes, ingestão de pequena quantidade de alimentos, baixa ingestão hídrica, dificuldade de deglutir dieta sólida e outras. A maioria desses problemas prevaleciam na alta hospitalar, embora alguns já estivessem amenizados, outros, se transformado como no caso de idosas que fizeram uso de sonda nasogástrica durante a hospitalização e foram para casa aceitando dieta por via oral com dificuldade e necessitando de ajuda dos familiares.

GRÁFICO 1- DISTRIBUIÇÃO PERCENTUAL DAS ALTERAÇÕES FUNCIONAIS APRESENTADAS PELAS IDOSAS DURANTEA HOSPITALIZAÇÃO E NAALTA HOSPITALAR

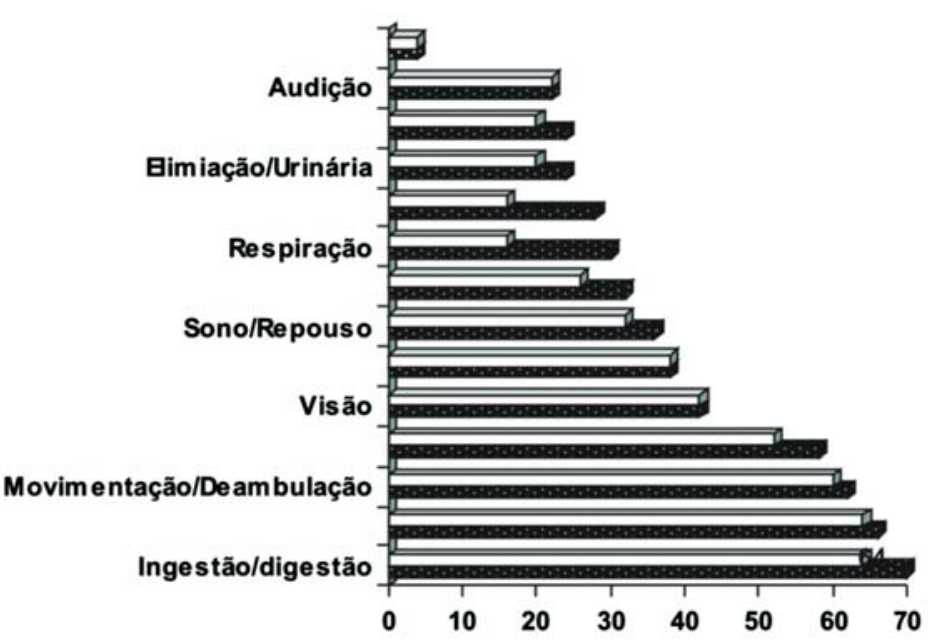

פ Durante internação\%

$\square \mathrm{Na}$ alta hospitalar\%

As inúmeras alterações que ocorrem no sistema digestivo do idoso (perdas dos dentes, diminuição do fluxo salivar e da peristalse esofagiana, alterações do paladar, redução da secreção ácida, diminuição da absorção dos nutrientes, entre outras) parecem justificar o surgimento de tais sinais e sintomas.

Em relação às alterações de pele/cabelos/unhas, essas se encontravam presentes em 33 (66\%) das mesmas. Foram detectadas várias idosas com úlcera de pressão, incisão cirúrgica, ulceração em membros inferiores, prurido e eritema, pediculose, pele fina e com escoriações e pele ressecada, No momento da alta, a maioria dos problemas permaneciam e outros se modificaram. Houve paciente, por exemplo, que 
apresentou necrose em pé direito e, após ter-se submetido à amputação do membro, saiu de alta com uma incisão cirúrgica necessitando ainda de cuidados na residência. A pele ressecada, pediculose e escabiose, tratadas durante a internação, foram identificadas para a continuidade da assistência no domicílio, não só ao idoso, mas também de seus familiares no sentido de evitar novas infestações.

As alterações de pele do idoso têm representado importante fator de desconforto e mal estar. Entre elas a pele seca, comum no idoso, pode causar prurido, descamação e rachaduras, além de diminuir a habilidade da pele de realizar muitas de suas funções. Também a umidade presente em algumas regiões do seu corpo imobilizado como a axila, região inguinal e inframamária nos idosos do sexo feminino compromete a integridade da pele. Além disso, o confinamento no leito e/ou cadeira predispõe ao aparecimento de úlcera de pressão, complicação essa que demanda alto custo financeiro e representa grande sofrimento para o idoso (FRANTZ; GARDNER, 1994; KEMP; KROUSKOP, 1994).

As alterações de movimentação e deambulação estiveram presentes em 31 (62\%) das idosas durante a hospitalização e em 30 (60\%) na alta hospitalar. As idosas em estudo apresentaram tais alterações devido a hemiplegia por seqüela de AVC, fratura de fêmur, dor em membros inferiores, amputação de membro inferior, "rigidez de membros inferiores" e outros, levando à constatação de que se trata de alterações crônicas de difícil solução em pequenos períodos de tempo. No entanto, são alterações que precisam ser consideradas e tratadas por representarem forte impacto na qualidade de vida dessas pessoas.

Entre as alterações mais freqüentes nas idosas em estudo, as alterações circulatórias apareceram em 29 (58\%) delas durante a internação e prevaleceram em 26 (52\%) na alta hospitalar. As mais freqüentes dessas alterações foram a hipertensão arterial, diminuição da perfusão periférica, arritmias, veias anormalmente dilatadas em membros inferiores, edema de membros inferiores, além de outras. Durante a hospitalização houve controle dessas alterações e redução da intensidade das mesmas, porém na alta hospitalar, elas ainda se faziam presentes, necessitando de controle adequado para a manutenção do bem-estar do idoso.

As alterações circulatórias têm grande implicação para o processo de educação em saúde realizada por profissionais, pois medidas como realização de atividades físicas, dieta hipossódica, redução do estresse, controle regular da pressão arterial, uso correto da medicação e controle do peso são essenciais na manutenção da sua saúde (SMELTZER; BARE, 1994).

Ao verificarem as alterações de visão, constatou-se que $21(42 \%)$ das idosas as apresentavam tanto durante a hospitalização como na alta. Entre elas, as mais encontradas foram diminuição da acuidade visual, retração palpebral, cegueira e secreção. Tais dados são concordantes com os achados de outros estudos (MONTESANTI et al. 1999).

As perdas sensoriais, segundo GRAVES (1988), começam na meia idade, evoluem progressivamente e causam limitações da atividade no indivíduo a ponto de, aos 70 anos, todos os sentidos apresentarem uma diminuição funcional.

Alterações de eliminação fecal estiveram presentes em 19 (38\%) das idosas durante a internação e em 17 (34\%) na alta hospitalar, sendo a mais freqüente delas a constipação intestinal, na maioria das vezes em condição crônica, inclusive com uso de laxante. A incontinência fecal e a diarréia também foram encontradas.

MACIEL e MELO (1997), lembram que a constipação intestinal, longe de ser um problema simples para o idoso, constitui-se em "importante causa de morbidade, piora a qualidade de vida e sentimento de bem estar".

Não chega a ser novidade que fatores como hábitos intestinais irregulares, uso indiscriminado de laxante, a falta de movimentação, dieta com poucas fibras e baixa ingestão de líquidos desencadeiam, ao longo da vida, a constipação intestinal. Assim, aos profissionais cabe um trabalho educativo em nível preventivo.

CASTLE (1989), lembra que, quando se trata de constipação no idoso, é importante investigar a época de início dos sintomas, pois se ele apresentar uma constipação ou tiver uma piora súbita da mesma é necessária uma investigação mais ampla para descartar complicações como carcinoma de cólon.

O sono e repouso representam fator importante na qualidade de vida dos idosos, interferindo no bem-estar, disposição e capacidade de realização das suas atividades do dia-a-dia. Essas alterações estiveram presentes em dezoito das idosas em estudo durante a hospitalização. Destas, apenas duas referiram que sua ocorrência se devia às interferências do ambiente hospitalar. Para 17 (34\%) das idosas tratava-se de alterações que as afetavam há algum tempo e que, segundo elas, estavam relacionadas com tristeza e preocupação, muitas vezes surgidas após uma perda significativa durante a vida.

Estudos têm demonstrado mudanças relativas ao sono, no idoso, quanto à continuidade, duração e profundidade. Os períodos de insônia tendem a ser mais freqüentes, o tempo de sono declina de 7 horas para 0 indivíduo de 33-45 anos para 06 horas no indivíduo dos 50 90 e a profundidade é afetada tanto em quantidade como em qualidade (MORGAN, 1993; FOREMAN; WYKLE, 1995).

A sensibilidade e regulação encontravam-se alteradas em 16 (32\%) das idosas durante a hospitalização e em 13 
(26\%) na alta hospitalar. A dor foi referida em diferentes regiões do corpo. Algumas se apresentavam gementes e outras, com dor ao serem manipuladas.

A dor no idoso é considerada um problema de alta prevalência, limitante de atividades e causadora de grande sofrimento, principalmente, por tratar-se, na maioria dos casos, de dor crônica, a qual acompanha o idoso por longos períodos de tempo. Quanto à sua prevalência, é considerada três vezes mais freqüente nos idosos ao comparar com pessoas de menos idade. Em idosos que vivem na comunidade, a dor é referida por 25 a 50\% deles, em idosos institucionalizados chega-se a verificar índices de 85\%. (FULMER et al., 1996).

Assim, o alívio da dor representa um importante aspecto na qualidade de vida dessa pessoas, porém, notase uma tendência a subestimá-la e a considerá-la como uma alteração própria do processo de envelhecimento. Ao atuar com esses idosos, é preciso desenvolver estratégias adequadas de assistência e, sendo a terapêutica medicamentosa a mais utilizada atualmente, os seus efeitos colaterais necessitam de monitoramento e controle rigoroso, devido às modificações que o idoso apresenta em nível de absorção, metabolismo, distribuição e excreção das drogas. Além disso, outras alternativas de assistência como o relaxamento, distração, música, meditação e aplicação de calor e frio podem ser utilizadas.

Quanto às alterações de eliminação/secreção, 14 (28\%) das idosas as apresentavam durante a hospitalização: secreção serosanguinolenta em ulcerações, dreno de tórax com secreção sanguinolenta, secreção em traqueostomia, secreção serosanguinolenta em incisão cirúrgica e outras. Das idosas em estudo, oito (16\%) ainda receberam alta com presença de secreção em ulcerações e/ou incisões.

Das idosas, 15 (30\%) apresentavam alterações respiratórias durante a hospitalização. Dessas, destacam-se a dispnéia e uso de cateter de $\mathrm{O} 2$, tosse produtiva, dispnéia aos esforços, cianose de extremidades e uso de traqueostomia. $\mathrm{Na}$ alta hospitalar, alguns desses problemas tinham sido solucionados e outros amenizados. Mesmo assim, oito idosas (16\%) continuaram apresentando algum tipo desses sinais e sintomas. A dispnéia aos esforços ocorre tanto pela sobrecarga circulatória como pela diminuição da eficiência respiratória. A tosse produtiva decorre freqüentemente dos processos infecciosos, aos quais, segundo ANZOLA PÉREZ (1993), o idoso está predisposto, principalmente, pela diminuição dos mecanismos de defesa (cílios, muco e reflexo da tosse).
As idosas em estudo também apresentaram alterações urinárias, sendo 12 (24\%) durante a hospitalização e dez (20\%) na alta hospitalar. A mais freqüente das alterações foi a incontinência urinária. Durante a hospitalização se resolveram alterações como a retenção urinária, hematúria e uso de sonda vesical de demora.

Outras conseqüências podem decorrer da incontinência urinária, tanto físicas como úlcera de decúbito e infecção urinária e conseqüências psicológicas desde perda da autoestima e isolamento social.

Mesmo considerando a alta prevalência e as conseqüências da incontinência urinária, os idosos não têm procurado tratamento específico para o problema e observase, na prática, diária que nem mesmo os profissionais parecem despertos para o problema.

Alterações do estado mental também foram encontradas em 12 (24\%) das idosas durante a hospitalização e em dez (20\%) na alta hospitalar. Ao considerar as causas de alterações cognitivas nos idosos, a Doença de Alzheimer não pode ser descartada devido à alta incidência entre tal população.

\section{CONSIDERAÇÕES FINAIS}

No presente estudo, foram incluídas 50 idosas, sendo 13 (26\%) pertencentes à faixa etária de 70 a 74 anos, 12 (24\%) dos 60 a 64 anos, dez (20\%) dos 65 a 69 ano e as demais encontravam-se com 75 anos ou mais. Entre elas, 35 (70\%) tinham boas condições de comunicação, 30 (60\%) eram analfabetas. Ficaram internadas de 6 a 10 dias, 22 (44\%) das idosas, dez (20\%) tiveram internadas num período de 11 a 15 dias. Apresentaram em média dois diagnósticos médicos e faziam uso de 2,6 medicamentos/idosa.

As alterações funcionais mais freqüentes, durante a hospitalização, eram as referentes à ingestão e digestão, presentes entre 35 (70\%) das idosas, alterações de pele/ cabelos/unhas estavam presentes em 33 (66\%) delas. Ainda, 31 (62\%) tinham alterações de movimentação e deambulação, 29 (58\%) alterações circulatórias, 21 (42\%) alterações de visão, 19 (38\%) alterações de eliminação fecal, entre outras como de sono e repouso, sensibilidade e regulação, eliminação/secreção, respiratória, urinária e de estado mental. A maioria dessas alterações permanecia no momento da alta hospitalar, algumas foram amenizadas e outras resolvidas durante a hospitalização.

Tais alterações, comuns no processo de envelhecimento pela diminuição da reserva funcional, exigem 
ações preventivas, curativas e de reabilitação, uma vez que existem inúmeras maneiras de aproveitar a capacidade do organismo com a finalidade de atenuar a dependência.

Aos idosos, não dispondo de recursos necessários para a manutenção da saúde e da vida, resta-lhes como possibilidade a assistência hospitalar, quando a situação se agrava. Como essa assistência demanda alto custo para sua operacionalização e atende apenas às necessidades de maior risco, aos idosos faltam recursos financeiros, programas de auxílio domiciliar e organização comunitária para seu atendimento.

No hospital os pacientes têm passado de forma rápida quando apresentam complicações agudas no seu estado de saúde. Os profissionais da saúde, por sua vez, não se encontram instrumentalizados para lidar com essa realidade e garantir a continuidade da assistência no domicílio que tanto se faz necessária, na medida que se deseja obter maior resolutividade dos problemas.

A alta precoce é uma possibilidade a ser considerada em nosso meio, uma vez que o sistema de saúde atual visa essencialmente a desospitalização. Com isso é preciso criar estratégias adequadas para contemplar o atendimento das necessidades da população.

Há necessidade, também, de assistência contínua e desenvolvimento de habilidades para o autocuidado tanto do idoso como dos familiares para reduzir limitações e dependências. Configura-se, então, a grande preocupação com essa população no presente momento.

Ao idoso são necessárias medidas que possibilitem a utilização do seu potencial, a realização de atividades diversas, estímulo à criatividade, de forma a manter o sentimento de utilidade e bem-estar. Caso contrário é possível que, dentro de pouco tempo, a situação se torne ainda mais crítica devido ao aumento crescente da população idosa.

ABSTRACT: Old people often present specific alterations, and they are vulnerable to multiple losses, especially on functions of the organism, making them susceptible to diseases and hospitalization. Considering that old people, when hospitalized, presented an acute health alteration, the low functional reserve makes it difficult for their recovering and that the care about those people must be continuous, in the present study functional alterations were identified in a group of 50 old women interned in a Medical Clinic of a General Hospital, during the hospitalization and at the hospital discharge. At the analysis of the results it was verified high rates at function's alteration, at the moment of admittance, many of which were just lessened during the hospitalization and the others have changed. Among the functional alterations presented by the old women the ones related to ingest/digest come off in 35 (70\%) of them during hospitalization and in $32(64 \%)$ at the moment of the hospital discharge, skin, nails and hair alterations in $33(66 \%)$ of the ladies during hospitalization and in $31(62 \%)$ at the moment of the discharge, body movement alteration in $31(62 \%)$ and $30(60 \%)$ of them during the hospitalization and at the discharge also, we can take other results as example like blood circulate alterations, vision, eliminatons, sleep and rest, sensibility, hearing and breathing. In addition to that, they presented an average of two medical diagnosis and were using 2,6 medicine/old women, making it clear the need of actions able to provide continuity to the complex assistance required for the prevention, healing and recovering of their health conditions.

KEY WORDS: Aged; Hospitalization; Patient's hospital discharge.

\section{REFERÊNCIAS}

1 ANZOLA PÉREZ, E. (Ed.). Enfermeria gerontológica: conceptos para la practica. Washington: OPAS, 1993.

2 BERQUÓ; LEITE, V.M. Algumas considerações sobre a demografia da população idosa no Brasil. [s.I.; s.n.], 1988

3 BURNSIDE, I.M. (Ed.). Enfermagem e os idosos. São Paulo: Andrei, 1979

4 CASTLE, S.C. Constipação: endêmica entre idosos?: gerontofisiologia, avaliação e tratamento. Clin. Med. Am. Norte, Rio de Janeiro, n.6, 1989. p. $1639-1652$

5 CHELALA, C.A. La salud de los ancianos: uma preocupacion de todos. Washington: OMS/OPAS,1992.

6 FOREMAN, M.D.; WYKLE, M. Nursing standart-of-practice protocol: sleep disturbances in elderly patients; alterations in the sleep-wake cycle call for immediate assessment and intervention. A NICHE project protocol. Geriatr. Nurs., New York, v.16, n.6, Sept./Out., 1995. p. 238-243.

7 FRANTZ, R.A.; GARDNER, S. Clinical concerns: management of dry skin. J Gerontol. Nurs. Therefore, v. 20, n.9, Sept., 1994. p.15-18.

8 FULMER, T.T.; MION, L.C.; BOTTRELL, M.M. Pain management protocol: inappropriate pain management leaves both the elder and the nurse feeling unfulfilled and unhappy with the care. Geriatr. Nurs., New York, v. 17, Sept./Oct., 1996. p. 222-227.

9 IBGE. Anuário estatístico do Brasil. Rio de Janeiro, 1991.

10 GOMES, F.A.A.; FERREIRA, P.C.A. Manual de geriatria e gerontologia. Rio de Janeiro: EBM, 1985

11 GRAVES, M. Physiologic changes. In: HOGSTEL, M.O. Nursing care of the older adult. 2.ed. Albany: Delmar, 1988. p.63-90.

12 GUTIERREZ GAMBARO, A. Atención y cuidado de enfermeria en el paciente senescente. Rev. Sanid. Def. Nac. (Santiago de Chile), Santiago, v.4, n.4, Sept./Oct., 1987. p. 366-371. 
13 KEMP, M.G.; KROUSKOP, T.A. Pressure ulcers: reducing incidence and severity by managing pressure. J. Gerontol. Nurs., Therefore, v.20, n.9, Sept.,1994. p. 27-34,52.

14 LESSA, I. Doenças crônico-degenerativas. In: ROUQUAYROL, M.Z. Epidemiologia e saúde. 3.ed. Rio de Janeiro: Medsi, 1990. p.411-420.

15 15. MACIEL, A.C.; MELO, M. Constipação intestinal no idoso. Gerontologia, São Paulo, v5, n.4, 1997. p.23-32.

16 MASLOW, A.H. Motivation and personality. 2.ed., New York: Harper \& Row, 1970.

17 MARIN, M.J.S. Levantamento de problemas dos pacientes idosos no momento da alta hospitalar. Dissertação (Mestrado) Escola de Enfermagem, Universidade de São Paulo. Ribeirão Preto, 1995. $130 \mathrm{f}$.

18 MONTESANTI, L.T. et al. Anamnese clínica x geriátrica. Gerontologia, São Paulo, v.7, n.1, 1999. p. 8-16.

19 MORGAN, K. Sleep in normal and pathological aging. In: BROCKLEHURST, J.C.; TALLIS,R.C.; FILLIT, H.M. Textbook to geriatric medicine and gerontology. 4.ed. Edinburg: Churchill Livingstone, 1993. p.122-128.
20 NERI, A.L. Qualidade de vida no adulto maduro: interpretações teóricas e evidencias de pesquisa. In: NERI, A.L. (org.). Qualidade de vida e idade madura. Campinas: Papirus, 1993, p.9-55.

21 SMELTZER, S.C.; BARE, B.G. Brunner/Suddarth tratado de enfermagem médico-cirúrgica. 7.ed. Rio de Janeiro: Guanabara Koogan, 1994. v.1, cap. 3, p.141-166.

22 TRIVIÑOS, A.H. Introdução à pesquisa em ciências sociais: a pesquisa qualitativa em educação. São Paulo: Atlas, 1987.

23 VERAS, R.P. País jovem com cabelos brancos: a saúde do idoso no Brasil. 2.ed. Rio de Janeiro: Relume Dumará, 1994.

Endereço do autor: Rua Filomena Otaiano Losasso, 50 - Jardim Ohara 17506-440 - Marília - São Paulo - Brasil Email: marnadia@terra.com.br

\section{ANEXO I - INSTRUMENTO DE COLETA DE DADOS}

\section{1 - DADOS DE IDENTIFICAÇÃO:}

Idade: Escolaridade

Condições de comunicação:

Quem responde:

Diagnóstico médico:

Número de internação no ultimo ano:

\section{2 - AVALIAÇÃO FUNCIONAL}

Medicação que faz uso:

Respiração:

Circulação:

Ingestão/digestão:

Eliminação urinária:

Eliminação fecal:

Eliminação secreção:

Pele/cabelos/unhas:

Sensibilidade/regulação:

Sentidos: visão audição tato/olfato/paladar -

Sono/repouso:

Movimentação/deambulação:

Estado mental: 